\title{
Detection of genetically modified soy in processed foods sold commercially in Malaysia by PCR-based method.
}

\begin{abstract}
Regulations for the use and labeling of genetically modified organism (GMO) products and derived ingredients are being implemented worldwide, that demands reliable and accurate methods to detect GMO in raw materials and food products. In this study, polymerase chain reaction (PCR) method was established for monitoring products derived from GMO that are sold in the markets in Malaysia, which specifically amplify the $35 \mathrm{~S}$ promoter, nos (nopaline synthase-terminator), EPSPS (5-enolpyruvylshikimate-3- phosphate synthase) and RRS (CTP/CP4EPSPS). Using this method, we investigated the incidence of genetically modified soy(GM-soy) and specifically the presence of roundup ready soy (RRS). All the soybean samples were evidenced by presence of the lectin gene. Out of 85 samples examined, the 18 positive GM samples were raw bean (9), tofu (8) and tempe (1) (a traditional Malayfood). The results demonstrate for the first time the presence of GM-soy in Malaysian food products, reinforcing the need for the development of accurate quantitative methods for routine analyses.
\end{abstract}

Keyword: Genetically modified organisms, Food products, Detection, PCR, Soy 